\title{
Exercise Intensities and Enjoyment Derived by Senior Short Tennis Players: Comparison of Game Effects with Opponents of Different Combined Skill Levels ${ }^{\dagger}$
}

\author{
Noboru HARADA $*$, Yuko OGUMA*,**, Hiroyuki ISHIDA*,** \\ and Takehiko SANO*
}

\begin{abstract}
PURPOSE : This study aims to verify the effect of differences in the skill level of opponents and doubles partners regarding exercise intensity and enjoyment of healthy senior people with no experience in playing tennis or short tennis when playing doubles games of short tennis.

METHODS : Twenty-four Japanese senior novices (52-77 years old; 8 males, 16 females) played four different types of doubles games, one game each against two different opponents (novice and skilled) and with two different partners (novice and skilled). Exercise intensity was assessed by acceleration and heart rate, measured by an ActivTracer ${ }^{\circledR}$ AC-301A (GMS), while enjoyment was evaluated using the Physical Activity Enjoyment Scale.

RESULTS : When playing against novice opponents, mean acceleration with a skilled partner $(130.3 \mathrm{mG})$ was significantly lower than with a novice partner $(138.1 \mathrm{mG})$, but when playing against skilled opponents, there was no significant difference between a skilled partner and a novice partner. When paired with a skilled partner, mean acceleration against skilled opponents (148.2 $\mathrm{mG}$ ) was significantly higher than against novice opponents $(130.3 \mathrm{mG})$, but when paired with a novice partner, there was no significant difference between skilled opponents and novice opponents. There was a significant main effect of the opponents for mean heart rate when playing against skilled opponents $(124.8$ beats/min) than against novice opponents (120.2 beats/min), but no significant main effect of partners was evident. There was no significant difference in enjoyment level among games.

CONCLUSION : This study demonstrates that when senior people with no experience start playing short tennis, they should carefully consider the skill level of their partners and opponents so that they can gradually increase their exercise intensity. The study also indicates how senior people can derive enjoyment from short tennis irrespective of the level of play of their opponents or doubles partners.
\end{abstract}

Key words : Intensity of Exercise, Acceleration, Enjoyment, Senior Novice Player, Short Tennis

\section{Introduction}

Short tennis was developed in Sweden in the 1970s as an introduction to tennis. The sport enables even children with minimal physical strength to engage in groundstroke rallies straightaway. A

\footnotetext{
${ }^{\dagger}$ Manuscript Received 25 September 2013, Accepted Publication 12 December 2013

中高齢初心者におけるショートテニス競技中の運動強度と楽しさの評価：対戦相手や味方パートナーのテニス技 量の異なるダブルスゲーム間の比較

${ }^{*}$ Graduate School of Health Management, Keio University, 4411, Endo, Fujisawa, Kanagawa, Japan (252-0883)

** Sports Medicine Research Center, Keio University, 4-1-1, Hiyoshi, Kohoku-ku, Yokohama, Kanagawa, Japan (223-8521)
} 
short tennis court is the same size as a badminton court (i.e., approximately one-third the size of a standard tennis court), and the ball used during play is lighter than a tennis ball and is made of sponge, meaning that its bounce and speed level is lower, whereas the racket is lighter and shorter than a standard tennis racket. Short tennis was devised to introduce children to tennis; thus, it is also thought to be suitable for elderly people with no experience playing tennis ${ }^{1)}$. Studies that have measured the exercise intensity of short tennis for elderly people have found that it generates less than or equal to $75 \%$ of the maximum heart rate (HRmax) in doubles games ${ }^{2) 3}$, suggesting that short tennis doubles games are suitable for elderly people. The results of these studies rely heavily on the HRmax estimation formula, 220 - age. However, the accuracy of the formula is less than desirable for exercise training intensities prescriptions ${ }^{4)}$.

In a study of exercise intensity during 30 minutes of ground-stroke rallies by university students playing short tennis, the mean heart rate was 130 \pm 9 beats/minute (BPM) in the skilled group and $159 \pm 10$ BPM in the novice group, with mean oxygen consumption (mean $\% \dot{\mathrm{V}} \mathrm{O}_{2} \max$ ) calculated on the basis of the heart rate being $46 \%$ in the skilled group and $68 \%$ in the novice group. Mean energy expenditure was calculated as $0.092 \pm 0.023$ $\mathrm{kcal} / \mathrm{kg} /$ minutes in the skilled group and $0.119 \pm$ $0.019 \mathrm{kcal} / \mathrm{kg} /$ minutes in the novice group, with the novice group engaging in moderately intense exercise at a significantly higher intensity than the skilled group ${ }^{5)}$. However, no investigation was made on the intensities of singles or doubles games which are quite different from the setting of 30 minutes ground-stroke rallies.

Exercise intensity was measured for one healthy young adult during singles and doubles games of short tennis (unpublished data). After $\dot{\mathrm{V}}_{2}$ max was recorded, a $\mathrm{K}_{4} \mathrm{~b}^{2}$ portable expiration gas analyzer (COSMED, Rome, Italy) and an ActivTracer ${ }^{\circledR} \mathrm{AC}^{-}$
301A three-dimensional accelerometer and pulse recorder (GMS, Tokyo, Japan) were used to measure oxygen uptake $\left(\dot{\mathrm{V}}_{2}\right)$ and body movement, respectively, to evaluate exercise intensity. The $\mathrm{K} 4 \mathrm{~b}^{2}$ and the ActivTtracer ${ }^{\circledR}$ have been validated against the criterion Douglas bag method ${ }^{6(7)}$. The $\mathrm{K} 4 \mathrm{~b}^{2}$ has been used for metabolic measurement on various types of physical activities ${ }^{8)}$ while the ActivTracer ${ }^{\circledR}$ can count movements in sedentary to vigorous activities and it can be used to predict energy expenditure and to classify levels of physical activity ${ }^{9}$. The results showed that during singles games, $\dot{\mathrm{V}} \mathrm{O}_{2}$ was $29.6 \mathrm{~mL} / \mathrm{min} / \mathrm{kg}, \% \dot{\mathrm{V}} \mathrm{O}_{2} \max$ was $66.5 \%$, and mean acceleration was $410 \mathrm{mG}$, whereas during doubles games, $\dot{\mathrm{V}} \mathrm{O}_{2}$ was 21.8 $\mathrm{mL} / \mathrm{min} / \mathrm{kg}, \% \dot{\mathrm{V}} \mathrm{O}_{2} \max$ was $52.1 \%$, and mean acceleration was $289 \mathrm{mG}$, suggesting that doubles games may qualify as a form of non-strenuous exercise for senior people. Exercise intensity was measured during 10 minutes of two different types of ground-stroke rallies (low-intensity rallies and high-intensity rallies) for one healthy young adult with high cardiopulmonary capacity and another with low capacity (unpublished data). For the high cardiopulmonary capacity individual, $\dot{\mathrm{V}}_{2}$ was $29.8 \mathrm{~mL} / \mathrm{min} / \mathrm{kg}$ and $43.4 \mathrm{~mL} / \mathrm{min} / \mathrm{kg}$, respectively; $\% \dot{\mathrm{V}} \mathrm{O}_{2}$ max was $56.8 \%$ and $80.9 \%$, respectively; and mean acceleration was $274 \mathrm{mG}$ and $507 \mathrm{mG}$, respectively; whereas for the low cardiopulmonary capacity individual, $\mathrm{V}_{2}$ was $27.4 \mathrm{~mL} / \mathrm{min} / \mathrm{kg}$ and $35.6 \mathrm{~mL} / \mathrm{min} / \mathrm{kg}$, respectively; $\% \dot{\mathrm{V}} \mathrm{O}_{2}$ max was $73.4 \%$ and $88.5 \%$, respectively; and mean acceleration was $253 \mathrm{mG}$ and $476 \mathrm{mG}$, respectively, suggesting that short tennis may provide a form of exercise that is consistent with increased cardiopulmonary capacity.

Exercise intensity was measured for four healthy subjects aged 50 to 65 who had never played tennis nor short tennis (unpublished data). Heart rate and acceleration of body movement were used as indicators of exercise intensity, and were measured using an ActivTracer ${ }^{\circledR}$. The subjects each played one 
singles and one doubles game. The results showed that the mean heart rate was $149.4 \pm 12.1 \mathrm{BPM}$ in singles games and 136.3 $\pm 11.1 \mathrm{BPM}$ in doubles games, and the mean synthetic acceleration during matches was $404.7 \pm 51.2 \mathrm{mG}$ in singles games and $351.0 \pm 81.5 \mathrm{mG}$ in doubles games, with both indicators being significantly lower in doubles games. In this study, which compares singles games and doubles games, mean acceleration did not decrease as much as the mean heart rate in some subjects during doubles games, in others, the mean acceleration did not decrease to the same extent as did the mean heart rate. This suggests that although doubles games may be a suitable activity for senior people, exercise intensity may vary with the skill level of the opponent or doubles partner.

When senior people play doubles games of short tennis in an everyday setting, their skills are not necessarily equivalent to those of their opponent or doubles partner. Novices should start playing games with low exercise intensity and move on to higher-intensity games over time ${ }^{10)}$, it is important to identify how the tennis skills of opponents and partners affect exercise intensity. Enjoyment is also an important factor that influences whether elderly people make time to engage in sport or exercise ${ }^{11) 12}$. It is important to identify the effect of the level of play of opponents and partners on the enjoyment of games of short tennis.

\section{Objective}

The aim of this study was to verify the effect of differences in the level of play of opponents and doubles partners on the exercise intensity and enjoyment of healthy senior people with no experience in playing tennis nor short tennis when playing doubles games of short tennis.

\section{Methods}

\subsection{Experimental procedure}

Each subject played four different types of doubles games, one game each against opponents with different levels of play (a pair of novices or a pair of skilled players) and with two different partners, (a novice partner and a skilled partner). The first pair to win 7 points won the game. Opponents and partners were instructed in advance to emphasize enjoyment and to keep their playing style constant. Plenty of rest time was allowed between games. As it was anticipated that the exercise intensity of doubles games would rise as opponents' skill levels increased, the order in which the games were played was arranged so as to start with the game expected to have the lowest exercise intensity (i.e., against weaker opponents).

The level of play of the opponents was evaluated in terms of the International Tennis Federation's International Tennis Number (ITN) in doubles (ITNd ${ }^{13)}$. The ITNd was calculated by subtracting the partner's ITN from the combined ITNs of the two opponents. The lower the ITNd, the higher was the anticipated exercise intensity, and the order of games was therefore determined in terms of the highest ITNd score.

The ITN is used by the International Tennis Federation as an index of a player's general level of play ${ }^{14)}$. ITN 1 is used to designate elite players; ITN 2, 3, or 4, advanced players; ITN 5, 6, or 7, intermediate players; ITN 8,9 , or 10 , recreational players; and ITN 10.1, 10.2, or 10.3, novice players. In this study, ITN 10.1, 10.2, and 10.3 were all regarded as equivalent to ITN 10 .

Table 1 lists the levels of play of opponents and partners, and the playing order of games.

\subsection{Venue}

The experiments were carried out in an indoor tennis court to avoid the effects of weather and wind.

\subsection{Period}

The study lasted 3 months from October, 2011 to 
Table 1 The Tennis Level of Doubles Partner and Opponent, and the Playing Order of Game ( $n=24$ for each game)

\begin{tabular}{cccc}
\hline $\begin{array}{c}\text { Playing } \\
\text { Order }\end{array}$ & $\begin{array}{c}\text { ITN Rating } \\
\text { of Partner }\end{array}$ & $\begin{array}{c}\text { ITN Rating } \\
\text { of Opponents }\end{array}$ & $\begin{array}{c}\text { ITN double } \\
\text { of Opponents }\end{array}$ \\
\hline Game 1 & 3 & 10,10 & 17 \\
Game 2 & 10 & 10,10 & 10 \\
Game 3 & 3 & 4,7 & 8 \\
Game 4 & 10 & 4,7 & 1 \\
\hline
\end{tabular}

ITN doubles (ITNd) is defined by adding together the ITNs of the two opponents and subtract the ITN of partner. When ITNd is larger than ITN 10 such as Game 1 , the skill level of opponents is lower than subject's pair. When ITNd is smaller than ITN 10 such as Game 3 or Game 4, the skill level of opponents is higher than subject's pair.

December, 2011.

\subsection{Subjects}

Subjects were 24 healthy senior people ( 8 men, 16 women), aged 52 to 77 , with no previous experience playing tennis. In this study, we excluded individuals who met the following criteria: 1. an individual for who exercise was medically contraindicated (identified by a questionnaire such as the Physical Activity Readiness Questionnaire) ; 2. an individual who did not undergo regular medical examinations (at least once a year); 3 . an individual with a history or receiving treatment for heart disease or cerebrovascular disease; 4 . patients with diabetes mellitus, hypertension, or lipid disorders with no permission to exercise from their primary care physician. The subjects were recruited after responding to posters or leaflets at a facility managed by a public interest corporation that provides health and medical services to the general public.

\subsection{Evaluation parameters}

\subsubsection{Evaluation of basic attributes of subjects}

Basic attributes were evaluated in terms of the body mass index (BMI) and everyday levels of physical activity. Everyday physical activity was evaluated using the Japanese short version of International Physical Activity Questionnaire (IPAQ) ${ }^{15)}$ to measure how many days and how much time the subjects spend on vigorous activities (heavy lifting, or aerobics etc.), moderate activities (bicycling at a regular pace, or doubles tennis etc.), and walking during last 7 days. These were then converted into metabolic equivalents (METs) hour per week by using the Physical Activity Index (PAI). Total score of MET*hour/week calculated from PAI is classified into three levels (Low, Moderate, or High) of physical activity.

\subsubsection{Evaluation of exercise intensity}

Heart rate and values of synthetic three-dimensional (up-down, side-to-side, and forward-to-back), acceleration of body movement were used as indices of exercise intensity when playing doubles games. Measurements were made using an ActivTracer ${ }^{\circledR}$ AC-301A three-dimensional accelerometer and pulse recorder. The main unit of the device was attached to the small of the back, and heartbeat monitor electrodes were applied to three points on the front of the chest. Heart rate and acceleration were measured at 0.05 second intervals, with the main unit set to record the mean value for each second. Because the ActivTracer ${ }^{\circledR}$ accelerometer measures up-down, side-to-side, and forwardto-back acceleration of body movement, it is also capable of handling non-constant movement, and it is therefore used to measure physical activity levels during various types of recreational activity and housework ${ }^{16)}$. Heart rate and three-dimensional acceleration of body movement were evaluated in terms of mean values from the start to the end of games.

\subsubsection{Evaluation of mean acceleration in terms of METs}

Regression equation for $\dot{\mathrm{V}}_{2}$ and acceleration of body movement was calculated based on data 
obtained from the author's short tennis experiments on a single healthy young individual (Equation [1]). Then the $\dot{\mathrm{VO}}_{2}$ to METs were converted for evaluation (Equation [2] $)^{17)}$.

$$
\begin{aligned}
& \dot{\mathrm{VO}}_{2}=0.056 * \text { mean acceleration }(\mathrm{mG})+10.673 \\
& \left.\left(r^{2} \text { (adj. }\right)=0.617, p<0.05\right) \\
& \text { METs }=(3.5)^{-1} * \dot{\mathrm{VO}}_{2}
\end{aligned}
$$

\subsubsection{Evaluation of enjoyment}

Enjoyment of doubles games was evaluated according to the score calculated from the Japanese version of the Physical Activity Enjoyment Scale (PACES) (7-point continuum, 18 items) ${ }^{18)}$. PACES 18-items includes questions such as "It's no fun at all $(+1)$; It's a lot of fun $(+7)$ ", or "It's very refreshing $(+7)$; It's not at all refreshing (+1)". Scores from these questions are added. Higher score indicates higher level of enjoyment. Subjects completed the PACES immediately after the end of each game. Both the reliability of the PACES and its validity across exercise and sporting activities for which enjoyment can be anticipated to vary have been established ${ }^{19) 20)}$. It is also frequently used as a scale for investigating the relationship between physical activity and enjoyable feelings in health behavior theory ${ }^{2122)}$.

\subsubsection{Subjects' performance}

All games were video recorded. The time taken for each game, the number of strokes hit between each pair of subjects in each game, the number of strokes returned by each subject in each game, and the winners and losers were recorded while reviewing the video.

\subsection{Statistical analysis}

Heart rate, three-dimensional acceleration of body movement, and enjoyment of physical activity were statistically analyzed as follows. For normal data, two-factor analysis of variance with the partner's level of play and the opponents' level of play as factors was performed (correspondence present/present), and a Friedman test was used for non-normal data. In both cases, a multiple comparison was performed if a significant difference was found. The statistical software used was IBM SPSS Statistics version 19.0, and $p<0.05$ was regarded as significant.

\subsection{Excluded data}

If an arrhythmia was evident, this information was excluded from the heart rate analysis.

\subsection{Ethical considerations}

This study was approved by the Ethics Committee of the Graduate School of Health Management, Keio University.

\section{Results}

\subsection{Basic attributes of subjects}

Table 2 lists the age, BMI, and daily physical activity levels of the study participants. The level of tennis skills of all subjects was at the Starter Level.

\subsection{Subjects' performance}

Table 3 presents the subjects' performances $(n=21)$ in the four different types of doubles games. Data for three subjects for whom video recording failed are not included.

\subsection{Variation in heart rate and acceleration during games}

Fig. 1 and Fig. 2 show variations in the heart rate and acceleration of a 68-year-old woman during Game 4 (game against skilled opponents with a skilled partner) as one example.

\subsection{Heart rate}

Arrhythmia occurred prominently in two of the 24 subjects, and the data for these two subjects were excluded from the analysis. The data for the remaining subjects $(n=22)$ were normal and were therefore analyzed by two-factor analysis of variance. The results showed a significant main 
Table 2 Profile of Subjects $(n=24)$

\begin{tabular}{lcccc}
\hline & Mean & SD & Min & Max \\
\hline Age (years old) & 64.0 & 4.8 & 52 & 77 \\
BMI & 22.5 & 2.2 & 18.6 & 28.5 \\
Vigorous Activities (METs*hours/week) & 0.9 & 3.5 & 0 & 16 \\
Moderate Activities (METs*hours/week) & 10.4 & 13.3 & 0 & 48 \\
Walking (METs*hours/week) & 31.0 & 27.8 & 0 & 139 \\
\hline
\end{tabular}

Physical Activity Index (METs*hours/week) was calculated as follows: Vigorous Activities $=8.0 *$ vigorous-intensity activity hours * vigorous-intensity days; Moderate Activities $=4.0 *$ moderate-intensity activity hours $*$ moderate days; Walking $=3.3 *$ walking hours $*$ walking days

Table 3 Subject's Performance $(n=21)$

\begin{tabular}{lcccc}
\hline & Game 1 & Game 2 & Game 3 & Game 4 \\
\hline Opponent & Novice & Novice & Skilled & Skilled \\
Partner & Skilled & Novice & Skilled & Novice \\
\hline Duration of Game (sec.) & $242.9 \pm 51.1$ & $281.4 \pm 67.7$ & $299.6 \pm 81.2$ & $305.7 \pm 92.5$ \\
Stroke Agst Subject's Pair (hits) & $27.9 \pm 7.8$ & $29.9 \pm 10.6$ & $45.8 \pm 16.9$ & $43.6 \pm 19.6$ \\
Stroke Returned by Subject (hits) & $13.4 \pm 4.0$ & $14.5 \pm 6.5$ & $22.1 \pm 9.1$ & $19.3 \pm 7.7$ \\
Winning Rate & $66.7 \%$ & $42.9 \%$ & $28.6 \%$ & $9.5 \%$ \\
\hline
\end{tabular}

Data for three subjects for whom video recording failed are not included.

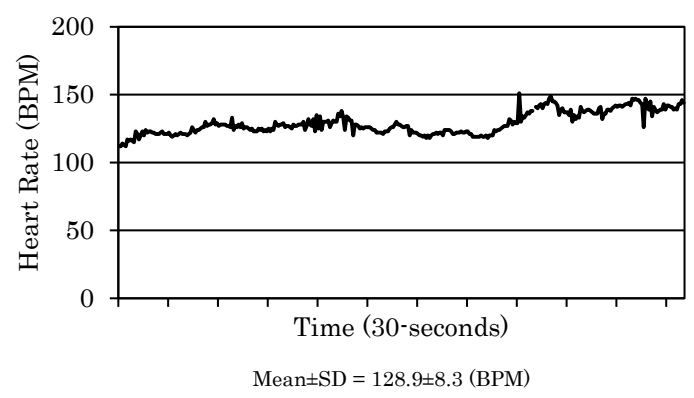

Fig. 1 Variation of Heart Rate of 68-year-old Female during Game 4

effect for the opponents, with the mean heart rate when playing a skilled pair of opponents (124.8 BPM) significantly higher than the mean heart rate when playing against a novice pair of opponents $(120.2 \mathrm{BPM})(F=7.684, d f=1, p=0.011$, partial $\left.\eta^{2}=0.268\right)$. There was no significant main effect of the partner. Fig. 3 shows mean heart rates during the four types of doubles games.

\subsection{Exercise intensity in terms of acceleration}

The data of subjects $(n=24)$ was analyzed

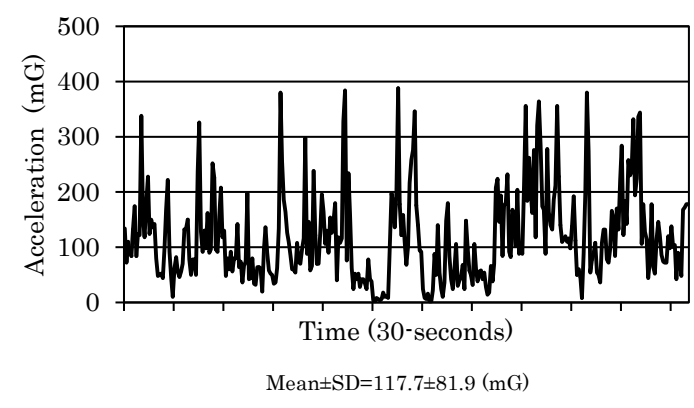

Fig. 2 Variation of Acceleration of 68-year-old Female during Game 4

by two-factor analysis of variance. The results showed a significant interaction $(F=6.323, d f=$ $1, p=0.019$, partial $\left.\eta^{2}=0.216\right)$. Testing of the simple main effects at each level showed that when playing against a novice pair of opponents, mean acceleration when paired with a skilled partner (130.3 mG) was significantly lower than the mean acceleration when paired with a novice partner (138.1 mG). When playing against a skilled pair of opponents, there was no significant difference between a skilled partner and a novice partner. When 


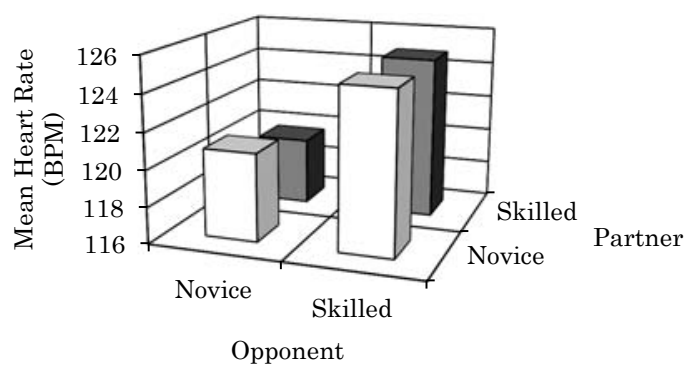

Mean Heart Rate was 119.7 BPM with a skilled partner against a novice pair of opponents, 120.8 BPM with a novice partner against a novice pair of opponents, 124.9 BPM with a skilled partner against a skilled pair of opponents, 124.8 BPM with a novice partner against a skilled pair of opponents.

Fig. 3 Mean Heart Rate during Playing against Different Skill-level of Opponents with Different Skill-level of Partners

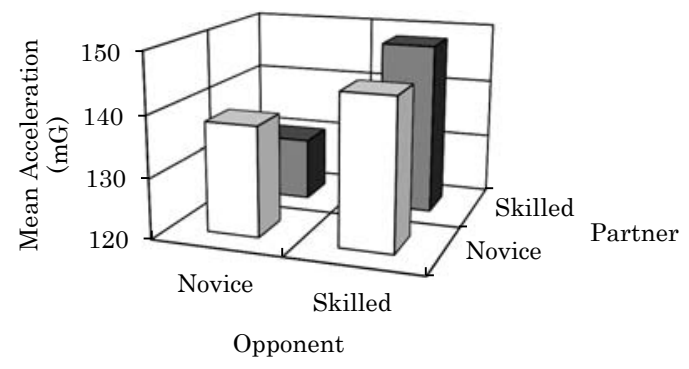

Mean Acceleration was $130.3 \mathrm{mG}$ with a skilled partner against a novice pair of opponents, $138.1 \mathrm{mG}$ with a novice partner against a novice pair of opponents, $148.2 \mathrm{mG}$ with a skilled partner against a skilled pair of opponents, $144.5 \mathrm{mG}$ with a novice partner against a skilled pair of opponents.

Fig. 4 Mean Acceleration during Playing against Different Skill-level of Opponents with Different Skill-level of Partners

paired with a skilled partner, mean acceleration when playing against a skilled pair of opponents (148.2 $\mathrm{mG}$ ) was significantly higher than the mean acceleration when playing against a novice pair of opponents $(130.3 \mathrm{mG})$. When paired with a novice partner, there was no significant difference between a skilled pair and a novice pair of opponents. Fig. 4 shows mean accelerations during the four types of doubles games.

\subsection{Exercise intensity in terms of METs}

When playing against a novice pair of opponents,

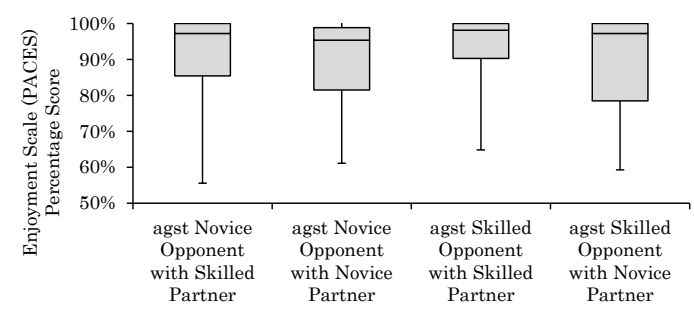

Top of box: 75 percentile, Middle of box: Median, Bottom of box: 25 percentile, Upper Whisker: Highest Score, Lower Whisker: Lowest Score

\section{Fig. 5 Enjoyment Scale (PACES) Percentage Score for Each Doubles Game}

there was a mean acceleration of $130.3 \mathrm{mG}$ (equivalent to 5.1 METs) when paired with a skilled partner, and a mean acceleration of 138.1 $\mathrm{mG}$ (equivalent to 5.3 METs) when paired with a novice partner. When playing against a skilled pair of opponents, there was a mean acceleration of $148.2 \mathrm{mG}$ (equivalent to 5.4 METs) when paired with a skilled partner, and a mean acceleration of $144.5 \mathrm{mG}$ (equivalent to 5.4 METs) when paired with a novice partner.

\subsection{Enjoyment}

The data was non-normal and was therefore analyzed by a Friedman test. Results indicated that there was no significant difference in PACES scores between doubles games. Fig. 5 shows a boxplot of PACES scores for doubles games. These scores have been converted into percentages.

\section{Discussion}

Exercise intensity in doubles games of short tennis played by senior novice players was affected by the level of play of opponents and partners. This study shows that senior novices, particularly those with low levels of cardiopulmonary fitness, can play comfortably if they choose to play in pairings that entail low exercise intensity and avoid those that entail high exercise intensity until they have become used to playing short tennis.

The level of enjoyment of doubles games was 
high in all games, irrespective of the level of play of either opponents or partners. The fact that the degree of exercise intensity does not affect enjoyment by senior novices indicates that they can enjoy playing in combinations chosen with an eye toward exercise intensity.

Exercise intensity during doubles games was analyzed by using mean values of heart rate and acceleration (Fig. 1 and Fig. 2). Heart rate gradually increased as the game proceeded, but the rate of increase was gentle, indicating that the subject was able to play comfortably (Fig. 1). Acceleration varied widely and continuously throughout the game, reflecting the repeated running and stopping movements required by short tennis. The waveforms for heart rate and acceleration indicated that although the subjects were active throughout the games, there was no increase in heart rate at times when their acceleration decreased, indicating that they were not overloaded during the games.

A comparison of exercise intensity during games in terms of mean heart rate showed that mean heart rate was higher when playing against a pair of skilled opponents compared with a pair of novice opponents, regardless of whether the doubles partner was a skilled player or a novice, and that the mean difference was 4.6 BPM. As shown in Table 3, when the opponents were skilled, they returned a greater number of strokes than when the opponents were a novice pair. This is a natural outcome of the difference in levels of play between skilled and novice players, but it also results in the senior novices themselves returning a greater number of strokes, games that last longer, and a higher mean heart rate.

A comparison of exercise intensity during games in terms of mean acceleration showed that when playing against a novice pair of opponents, mean acceleration was significantly lower when paired with a skilled player than with a novice player, and that the mean difference was $7.8 \mathrm{mG}$, equivalent to a difference of 0.2 METs. In short tennis, in principle, each player defends half of their own side of the court, but in games when subjects were paired with a skilled player, they may have tended to depend on them, leading to their defending a smaller area and reducing the level of exercise, and ultimately resulting in lower exercise intensity. Acceleration is known to have a close correlation with energy consumption during walking and running ${ }^{7 / 9)}$.

When paired with a skilled partner, mean acceleration was significantly higher when playing against a skilled pair of opponents compared with a novice pair, yielding a mean difference of $17.9 \mathrm{mG}$, equivalent to 0.3 METs. This is inferred from the fact that when playing together with a skilled player, there is a low likelihood of either the subject's partner or the opponents missing a stroke, meaning that games last longer and the extent of movement is also widened.

These results suggest that when senior novices, particularly those with low cardiopulmonary fitness, start playing short tennis, their exercise intensity will be higher if their opponents are highly skilled players, and it is then necessary that they be careful to avoid overexertion.

There was no significant difference in PACES scores between different combinations of opponents and partners with different levels of play; scores were high for all four different combinations, with a ceiling effect evident. There was also no correlation with performance in terms of factors such as results and number of strokes returned, as shown in Table 3. This suggests that senior novices derive great enjoyment from playing doubles games of short tennis. The fact that the number of people reporting low PACES scores tended to decrease as the number of strokes returned during a match increased, also suggests that physical movement and participation in the game may be an important factor in enjoyment.

This study includes two novel points. First, 
differences in the level of play of opponents or doubles partners have an effect on exercise intensity. Second, quantitative proof is offered that differences in the level of play of opponents or doubles partners have no effect on feelings of enjoyment.

This study has some limitations that must be noted. First, the study population included a large number of subjects who commonly exercised. It is therefore important to be cautious in applying the results to senior people who are not accustomed to exercise.

The second limitation is the effect of playing the games in a fixed order. In this study, the four doubles games began with the game assumed to entail the lowest exercise intensity. Despite the fact that plenty of rest time was allowed between games, there is a possibility that the order in which the games were played affected the results.

Regarding the third limitation, because the three beginners who were study collaborators participated in 48 games, we cannot exclude the possibility that this experience improved their level of play and consequently influenced the results. In this regard, however, when we measured the ITN scores of these three study collaborators again on the last experimental day, their scores increased from 58.3 \pm 18.7 points before the experiment to $59.9 \pm 20.8$ points after the experiment, which does not confirm a statistically significant difference. In addition, none of the collaborators achieved an ITN score of 75 points, which represents the recreational level. Therefore, their skill levels as measured by the ITN scores remained constant through a period of 3 months. On the other hand, the study subjects had limited opportunities to improve their performance skills with only 4 games.

The fourth limitation is a sample size problem. In a preliminary experiment, the efficacy amount was calculated based on the data from three singles game participants and one doubles game participant for who exercise intensity changed greatly compared with that of their corresponding opponents. Considering this, as well as the possibility of dropouts, we recruited a minimum of 20 subjects. However, in this study, the efficacy amount was not as large as the one in the preliminary experiment. Therefore, a larger sample size would be desirable in future experiments.

The fifth limitation is that the accelerometer was fixed to the small of the back. Short tennis is a racket sport that involves vigorous upper body movement. An accelerometer fixed to the small of the back is able to measure movement about the court, but is not capable of properly measuring upper body movement. The rackets used in short tennis are light and short, meaning that their effect on exercise intensity overall is not significant. Future investigations must consider the best way to measure upper body movement in order to enable more accurate evaluations (e.g., whether accelerometers should be worn on the upper arm of the dominant hand or around the wrist $)^{23)}$.

The sixth limitation is that the Japanese version of the PACES was used as a scale to measure enjoyment. A ceiling effect was evident in all four types of doubles games, and it is possible that this study may not have succeeded in confirming how the level of play of opponents or doubles partners affects enjoyment during play.

The seventh limitation is that factors affecting enjoyment other than the level of play of opponents or doubles partners were not assessed. The experiment was performed on an indoor court, successfully controlling the effects of weather and temperature on feelings of enjoyment, but the effect of factors such as how doubles partners addressed their subjects and how they behaved could not be regulated. Nevertheless, even after taking these limitations into consideration, short tennis may be regarded as an enjoyable activity for senior novices.

Considering the above-mentioned limitations, we want to examine the influences of different 
combinations in doubles games on the exercise intensity of the upper and lower limbs and the enjoyment of a population with no exercise habits, using a larger sample size and by randomizing the order in which the game is played.

\section{Conclusion}

The aim of this study was to verify the effect of differences in the level of play of opponents and doubles partners on the exercise intensity and enjoyment of healthy senior people with no experience in playing tennis nor short tennis when playing doubles games of short tennis.

The subjects of the experiment were 24 senior individuals, aged 52 to 77 , who played four different types of doubles game once each: Game 1 with a skilled partner against a novice pair of opponents, Game 2 with a novice partner against a novice pair of opponents, Game 3 with a skilled partner against a skilled pair of opponents, and Game 4 with a novice partner against a skilled pair of opponents. Exercise intensity during the games was evaluated in terms of mean values for heart rate and acceleration, and enjoyment was evaluated in terms of the score for the Japanese version of the PACES, which was completed immediately after each game as a physical activity enjoyment scale.

With respect to heart rate, data for two subjects were excluded from the analysis because of arrhythmia, meaning that a full evaluation could not be carried out. An analysis of the remaining 22 subjects showed that there was a significant main effect of the opponents for mean heart rate when playing against a skilled pair of opponents (124.8 BPM) than when playing against a novice pair of opponents (120.2 BPM), but no significant main effect of partners was evident.

With respect to acceleration, a significant interaction was evident. When playing against a novice pair of opponents, mean acceleration when paired with a skilled partner (130.3 mG, 5.1 METs) was significantly lower than that when paired with a novice partner (138.1 mG, 5.3 METs), but when playing against a skilled pair of opponents, there was no significant difference between a skilled partner and a novice partner. When paired with a skilled partner, mean acceleration when playing against a skilled pair of opponents $(148.2 \mathrm{mG}, 5.4$ METs) was significantly higher than that when playing against a novice pair of opponents (130.3 $\mathrm{mG}, 5.1$ METs), but when paired with a novice partner, there was no significant difference between a skilled pair and a novice pair of opponents. With respect to enjoyment, a ceiling effect was evident for PACES scores, and there was no significant difference between any of the four different types of doubles games.

The incorporation of moderate physical activity into daily activities of the senior is important for them to lead a healthy lifestyle ${ }^{24)}$, and doubles games of short tennis may offer an effective method of increasing the level of physical activity for senior persons. This study demonstrates that the exercise intensity of doubles games of short tennis is affected by the level of play of opponents or doubles partners, and that when senior people with no experience start playing short tennis, they should carefully consider the skill level of their partners and opponents so that they gradually increase their exercise intensity. The study also indicates how senior people can derive enjoyment from short tennis irrespective of the level of play of their opponents or doubles partners.

\section{Acknowledgement}

This study was partially supported by the Keio Gijuku Academic Development Funds.

\section{References}

1 ) Sato, M., and Sato, M.; Short tennis bible [in Japanese], Tokyo, Short Tennis Production, p.1, 1995. 
2 ) Shionoya, A., and Horiuchi, S.; Fundamental study on short tennis for the elderly people based on the PWC measurement and vibration analysis of short tennis racket [in Japanese], Bull Nagaoka Univ Technol, Vol. 25, pp.113-118, 2003.

3 ) Kitamura, K, and Toriumi, K.; Heart Rate during "RIZUMU TAISO" and "FURESSYU TENISU" of Middle Aged and Elderly Woman [in Japanese], J Laboratory for Work Physiology, College of Liberal Arts, Toyama Univ, Vol. 25, No.2, pp.25-33, 1992.

4 ) Whaley, M. H., et al.; Predictors of over-and underachievement of age-predicted maximal heart rate, Med Sci Sports Exerc,Vol.24, pp.1173$1179,1992$.

5 ) Watanabe, K., and et al.; Exercise Intensity during a 30-minute Ground Stroke Rally using Short-tennis Racket and Ball: Comparison between Skilled and Novice Groups, J Educ Health Sci, Vol. 52, No. 4, pp.259-266, 2007.

6 ) McLaughlin J. E., et al.; Validation of the COSMED K4 $\mathrm{b}^{2}$ Portable Metabolic System, Int J Sports Med, Vol.22, No.4, pp.280-284, 2001.

7 ) Oguma, Y., et al.; Assessment of intensity and amount of physical activity using triaxial accelerometry [in Japanese], J Sports Med Keio Univ, pp.25-31, 1999.

8 ) Millet GP., et al.; Effects of drafting on energy expenditure in in-line skating, J Sports Med Phys Fitness, Vol.43, No.3, pp.285-290, 2003.

9 ) Tanaka, C., et al.; Triaxial Accelerometry for Assessment of Physical Activity in Young Children, Obesty, Vol. 15, No. 5, pp.1233-1241, 2007.

10) Nelson, M., et al.; Physical Activity and Public Health in Older Adults: Recommendation from the American College of Sports Medicine and the American Heart Association, Med Sci Sports Exercise, Vol. 39, No. 8, pp.1435-1445, 2007.

11) Kondo, K.; Exploring "Inequalities in Health": A large-scale social survey for care prevention in Japan [in Japanese], Tokyo, Igakushoin, pp.53-58, 2007.

12) Kolt, G., Driver, R. and Giles , L. C.; Why Older Australians Participate in Exercise and Sport, J Aging Physical Activ, Vol. 11, pp.185-198, 2004.
13) The Tennis Development Department of the International Tennis Federation; International Tennis Number Manual, pp.21, 2004.

14) Pluim M. B., et al.; Health benefits of tennis, Br. J. Sports Med., Vol. 41, pp.760-768, 2007.

15) Murase N., et al.; Validity and reliability of Japanese version of international physical activity questionnaire [in Japanese], J Health and Welf Stat, Vol.49, pp.1-9, 2002.

16) American College of Sports Medicine; ACSM's Guidelines for Exercise Testing and Prescription, $7^{\text {th }}$ ed., Philadelphia, Lippincott Williams \& Wilkins, pp.147-149, 2006.

17) American College of Sports Medicine; ASCM's Guidelines for Exercise Testing and Prescription, $7^{\text {th }}$ ed., Philadelphia, Lippincott Williams \& Wilkins, pp.291-293, 2006.

18) Marcus, B. and Forsyth, L. A.; Motivating people to be physically active, trans. Shimomitsu, K., Nakamura Y., and Oka, K., Tokyo, Taishukan Book, pp.71-73, 2006.

19) Kendzierski, D. and DeCarlo, K. J.; Physical Activity Enjoyment Scale: Two validation studies, J Sport Exercise Psychol, Vol. 13, No.1, pp.50-64, 1991.

20) Mullen, S. P., et al.; Measuring enjoyment of physical activity in older adults: Invariance of the physical activity enjoyment scale (paces) across groups and time, Int J Behav Nutr Phys Activ, Vol. 8, pp.103-112, 2011.

21) Singer, R. N., et al.; Handbook of Sport Psychology, $2^{\text {nd }}$ ed., New York, John Wiley \& Sons, Inc., pp.636-671, 2000.

22) Rhodes, R. E. , et al.; A review and Meta-Analysis of Affective Judgments and Physical Activity in Adult Populations, Ann Behav Med, Vol. 38, No. 3, pp.180-204, 2009.

23) Graves, L. E. F., et al.; The contribution of upper limb and total body movement to adolescents' energy expenditure whilst playing Nintendo Wii, Eur J Appl Physiol, Vol. 104, No. 4, pp. 617-623, 2008.

24) Aoyagi, Y. and Shephard, R. J.; Steps Per Day: The Road to Senior Health?, Sports Med, Vol. 39, No. 6, pp.423-438, 2009. 